\title{
Handling Illusive Text In Document To Improve Accuracy Of Plagiarism Detection Algorithm
}

\author{
Zahid Iqbal \\ University of Gujrat
}

\author{
Shakeeb Murtaza \\ University of Gujrat
}

\author{
Hamid Ayub \\ University of Gujrat
}

\begin{abstract}
Plagiarism Detection is being one of the challenging tasks in academic research world to ensure integrity/authenticity of a document. Currently, many efficient algorithms are available to sufficiently detect the plagiarism in a document. Pre-processing of a document typically remain a master key to achieve maximum stable goal. Although all algorithms, before checking plagiarism, initially perform some sort of pre-processing on documents and convert the document into a particular format like by removing whitespaces and all special characters, etc. In this paper, we focus on two possible techniques, which can be used for plagiarism, which existing plagiarism detection algorithms are omitting. First is replacing the white spaces with a hidden character with white colour (background colour) between consecutive words so apparently, they seem to be distinct words, but algorithm/computer will incorrectly consider them as a single word. So even a $100 \%$ copied statement would not be identified as plagiarised content. Second is hiding spam text behind images to falsely report maximum number of words count in a document but as they are hidden so human eye can't discover them and algorithm will consider them as some words resulting in less percentile score of the plagiarised document. Our proposed (preprocessing) technique can efficiently handle these two critical problems which results in improved accuracy and authenticity of plagiarism checking algorithms. We have compared performance of our algorithm considering these critical issues with other stateof-art algorithm (particularly with Turnitin) and our algorithm handles these issues efficiently.
\end{abstract}

Index Terms-Plagiarism Detection Algorithm, Invisible Text, Text Behind Images

\section{INTRODUCTION}

Plagiarism is an act of copying someone else work and claim that this content belongs to you. Plagiarism in research and other academic documents has inevitably been of great concern. Cheaters employ a lot of tricks to avoid getting caught by a duplicate copy checking mechanism. Some of these are repositioning of parts of the text, changing document (file) names, rewriting portions of the text and/or changing synonyms of various words in paragraphs. Because of this, plagiarism detection becoming quite challenging [1]. In order to check the originality of documents many advanced plagiarism detection service providers came to existence such as Turnitin, Noplag, Plagscan, Copyscape etc. They provide facility to check the ownership of the document and generate originality report. Almost all of them perform some initial steps (pre-processing of the document) on the input document and turn it into a particular format according to their algorithm's scheme for further processing. The pre-processing may involve removal of whitespaces, punctuation letters, symbols and special characters. Pre-processing is performed to separate out the characters and symbols. Typically, a document can contain three types of content.

$$
\begin{aligned}
& \text { - Text } \\
& \text { - Image } \\
& \text { - Source code }
\end{aligned}
$$

A. Text: Text is a type of data that is written in any native language like English, Urdu or Arabic, etc. The text documents consist of many rules of particular language in which that text is written. The rules additionally include the appropriate use of commas, apostrophe, dashes and full stops. B. Image: Image is pixel data. Till today it is most difficult data type to check the duplication. Because change in the particular attribute can differ the whole result. Images are included in documents as visual data and diagrams, etc. The authors use one trick to prevent/overcome the rate of plagiarism by hiding out the useless data under the images that increases the number of characters in a document resulting in lesser rate of plagiarism. C. Source Code: It is a scripted code drafted in a computer language with keywords and rules of that languages e.g. semicolons, brackets, and inverted commas, etc. Pre-processing of such documents is done by removing all keywords and syntax rules. The remaining text is used to check the duplication of documents. Majority of the algorithms separates the following properties in pre-processing steps from the document before checking plagiarism.

Majority of the algorithms separates the following properties in pre-processing steps from the document before checking plagiarism.

- Whitespaces

- Punctuation marks

- Mathematical Symbols

- Images

- Physical Style of the document (colors, sizes, headings, etc.)

\section{LitERATURE REVIEW}

Various algorithms have been proposed in recent years to check the plagiarism of the document after applying some preprocessing.

\section{A. Citation Order Analysis}

This method is based on bibliographic coupling. It is employed to detect citation that are written but not cited in the document. The primary advantage of this document is 
its bilingual feature (language independent) and it can detect idea plagiarism. But employing this method, we cannot detect plagiarism passages of limited length [2].

\section{String Matching Algorithm}

It detects the plagiarism by finding the longest common subsequence between two documents or uncover patterns against the values stored in the database. It's significantly slow algorithm because it compares all sub-string of a document with all available strings. [3].

\section{A. Karp-Rabin and Greedy String Tiling Matching Algorithm}

This method is employed to detect common string sequence between two or more documents. It can detect a string which is built up from smallest string from various documents. It ignores punctuations when it performs searching and matching between documents. It generates the fingerprint of all documents and generates its hash values using ASCII values of the character using Karp Robin Rolling Hash and this hash function generate same value for two same string. The advantage of the greedy string-matching algorithm is that its performance is not affected if there are ample number of strings build from small strings of various documents [4].

\section{B. Authorship Verification for Short Messages using Stylome- try}

By employing this method, we are capable to identify the original author of the content. Because writing style varies from person to person. We check the pattern of writing style and can determine whether the person who is checking its plagiarism is the original author of the document or not? [5].

\section{Winnowing Algorithm}

The winnowing algorithm generates fingerprints of a document and then create windows of given size and select smallest hash value from each window. After this it matches the hashes with the hash values stored in the database [6].

\section{Plagiarism Detection using Apache Lucene (AL) and co- sine similarity}

$\mathrm{AL}$ is an open-source program utilized for indexing. After employing the indexing, the cosine similarity test is used to check the plagiarism rate between two documents. AL allows to process bulk data and increase processing speed dramatically [7].

\section{E. Cosine Similarity with Pre-Processing:}

In [8], vector space model is proposed as shown in Fig. 1 following steps are performed in this method; firstly, some sort of pre-processing is applied on collected data then different vectors are made via unigram, bigram and trigram techniques List of trigrams against each document is created after applying cosine similarity measures methods and finally, Jaccard similarity measure is used to compare each tri-gram pair. This algorithm includes some limitations that it can only produces cosine value (plagiarism percentage) but can't highlight the plagiarised statements [8].

\section{F. MOSS Tool:}

MOSS implements an algorithm based on a function by dividing text into $\mathrm{k}$-grams, where a $\mathrm{k}$-gram is an adjacent substring of length $\mathrm{k}$. Individual hash is made for every $\mathrm{k}$ gram, and MOSS selects a subset of these hash values as the document's fingerprints. Matching these fingerprints helps in identifying similarity of the document. Similarity rate depends on number of fingerprints matched. [9].

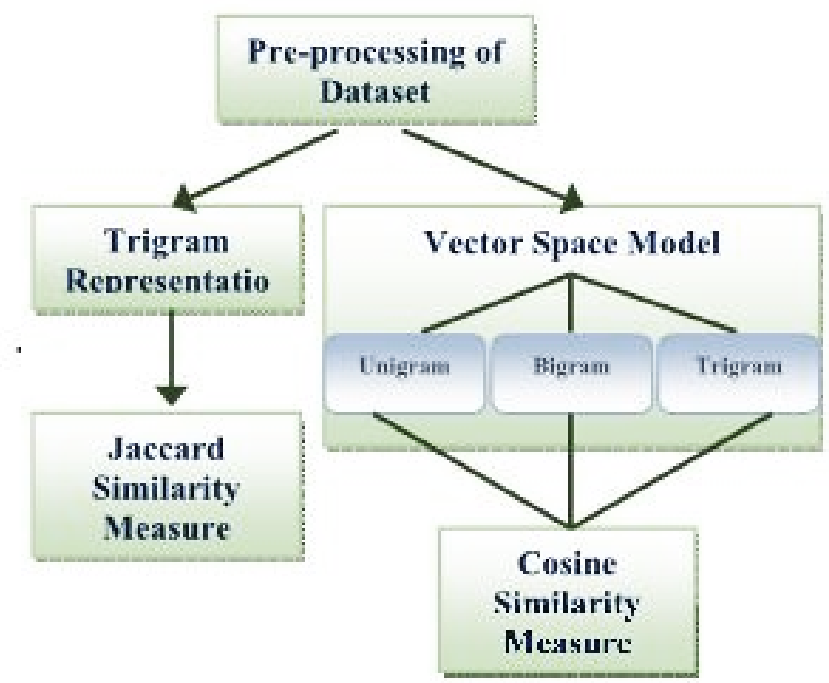

Fig. 1. Modular schema

In [10], a pre-processing method is proposed to ensure whether a document or paragraph is rephrased using synonyms or any document translator application. In the first place, it performs tokenization by converting the contents of the document into structured content, e.g. it separates punctuation marks for further processing. Subsequently, it eliminates such frequent words that has no meaning including lexical word such as 'is', 'are', 'am'. In lemmatization process, all words are converted in their base words like "does" to "do". Subsequently algorithm replaces all numbers.

with a dummy character to ensure the accuracy of plagiarism detection algorithm and in the last step (synonym recognition and word generalization), it tries to understand synonyms and generalize it to detect the translated paragraph as shown in Fig. 2.

In [11], the author proposed to do some sort of preprocessing, like removing comments \& blank spaces and using uniform tokens for primitive types \& variables, before applying actual process of intelligent matching techniques so that the tool can detect the copied code even if it is changed in format and variable names, etc.

\section{Problem Statement}

Though, different plagiarism detection algorithms are performing well and efficiently however, they nevertheless require improvements. Un-Ethical practices are being used to exploit the system and to obtain spurious report of originality. After 


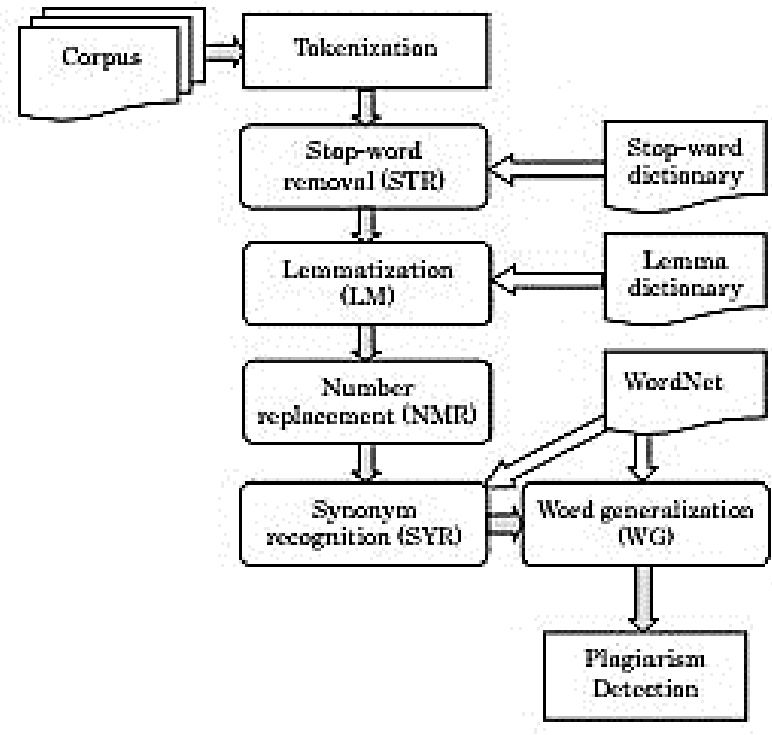

Fig. 1: Text pre-processing scheme

Fig. 2. Text Pre-Procesing [10]

performing a comprehensive literature review, we have identified two critical problems which, surprisingly, are not handled by most popular algorithms. First identified-problem is adding hidden characters (same colour as of the background) between two words. For example, if we look at the paragraph given in Fig. 3 apparently it looks like an ordinary paragraph, but reality is different.

\footnotetext{
"Hami is a bachelor student of computer science at university of gujrat. No doubt, He is pioneer student but he is too much careless which is seriously infecting his degree. We don't know what happen to his future if he performs the same."
}

Fig. 3. Text with hidden character(s)

In Fig. 4, realistic picture of matter can be seen. We have altered the colour of the whole paragraph as black in such way those illusive characters which were hidden among words by changing their colour as white colour (background colour), can be seen clearly. In this method, authors (cheaters) very smartly concatenate various words with such hidden words so that overall algorithms will consider them as a whole word and would be unable to check its plagiarism. Even such words possess no meaning but because almost all plagiarism detection algorithms don't check grammatical mistakes as they merely check the duplication so using this method, one can easily cheat the plagiarism detection algorithms.

The second critical problem which we have figure out is "hiding spam text behind the image to achieve maximum word count". The primary purpose of this cheat is to decrease

\begin{abstract}
"Hamisissabachelorsstudentsofacomputerasciencesatsuniversitys of gujrat. No doubt, $\mathrm{He}$ is pioneer student but he is too much careless which is seriously infecting hisadegree. We don't know what happen to hissfuture.ifahe performs the same."
\end{abstract}

Fig. 4. Text after revealing the hidden character(s)

plagiarism percentage value because algorithms counts the plagiarism in percentage so if we hide some garbage text behind images then this hidden garbage text will help to decrease the overall plagiarism percentage. For example, if we look at Fig. 5, it apparently shows that it is clearly showing a graph of some study, but it is not solely a graph rather we have hidden some spam text behind this image.

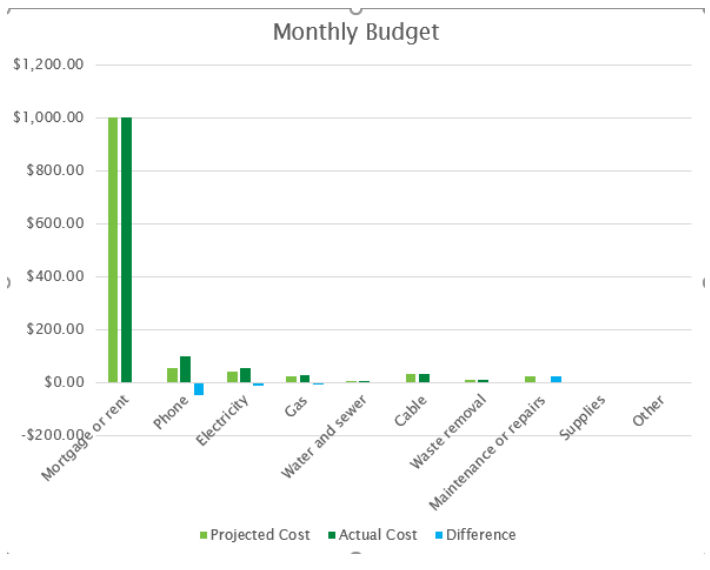

Fig. 5. Text behind the Image

In Fig. 6, we are showing that spam text which was hidden in the background of image.

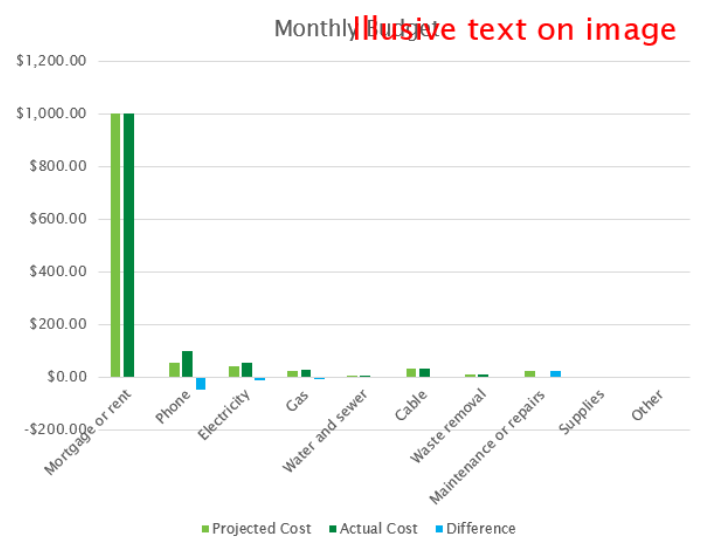

Fig. 6. Text visible on Image

It can be clearly perceived that the text over the image is useless, but it will increase the word count therefore, it will help to reduce the plagiarism percentage. 


\section{Proposed TeChnique}

In this paper, we have proposed a novel technique that would be applied on document as a pre-processing, before applying the actual algorithm of plagiarism detection, to improve the accuracy of plagiarism detection. The proposed algorithm also examines document properties (text-colour, image $\&$ text location, etc.) rather than relying on the text of the document. It checks the document properties to ensure that the document content is not illusive/masked (it is same as its appearance). After examining the document properties and removing the illusive content, our algorithm separates the text from the document for detecting the plagiarism in it.

We have identified two critical problems that helps the authors (cheaters) to cheat the plagiarism detection algorithm. First technique that is used to exploit the plagiarism detection system is including character of white colour (background colour). So, hidden character helps in the concatenation of two words and algorithm consider them as a single word so, apparently, these words look like an ordinary word.

Second technique that is used to cheat the algorithm is adding spam text behind the image that is not visible to the evaluator/supervisor. Spam text help to increase the word count of a document and also decrease the plagiarism percentage.

We divided illusive content detection algorithm into two parts. In the first part we detect and eliminate the invisible character that helps in the concatenation of two words to produce an unfamiliar word. In the second step, we detect and show the spam text that is hidden behind the images to increase word count and decrease plagiarism percentage.

To address the problem of invisible character, we proposed two methods. In first method, our algorithm read the document (MS Word document) XML and detects the background colour of each section in the document. After checking the background colour of each section, we iterate over each character of the document and check that the colour of the character is approximately same (not distinguishable by human) as the colour of background colour of the section. If we detect such character, then we will replace this character with "space". We are replacing such character because it possesses no meaning in the content, its scarcely added to cheat the plagiarism detection algorithm. There is an alternative method to handle this problem i.e. to examine each word in the dictionary, if it does not exist in the dictionary then it will be handled by the above stated algorithm. Although, this method can also be employed but it may not be so efficient as it can cost too much. To identify spam text behind the image, our proposed algorithm iterates on all images available in the document and check if there is any text behind the image. If the algorithm detects any illusive text behind the images, it brings the text in front of the image. Therefore, this text will be seen by the evaluators of the document. Proposed technique is also explained as flow chart in Fig. 7.

\section{RESULTS AND DisCUSSIONS}

We have compared our technique with currently present state of art techniques. The most popular and highly demanded

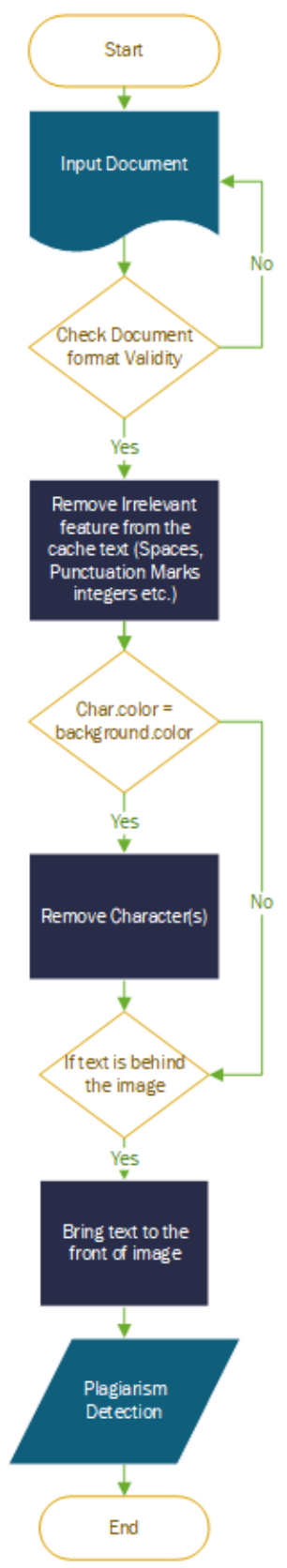

Fig. 7. Block Diagram

tool till now is Turnitin. Initially, we have checked the invisible character problem i.e. we copied text from Wikipedia and perform plagiarism test on it using Turnitin. As expected, we got the $100 \%$ plagiarism rate of the text that we have copied from Wikipedia as shown in Fig. 8. Fig 8 clearly shows that the content was $100 \%$ plagiarised and taken from Wikipedia. But when we applied above mentioned cheating technique 1 (invisible character to concatenate words) on some sentences of the paragraph (at starting and ending), the result was surprisingly different. The plagiarism rate reduces from $100 \%$ to $58 \%$ as shown in Fig 9. But it can be reduced to $0 \%$ 


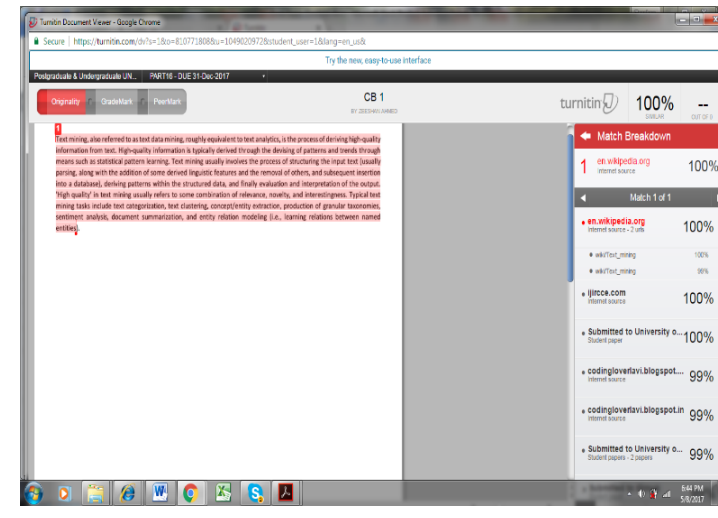

Fig. 8. Report without invisible characters

by employing this technique to all sentences of the document.

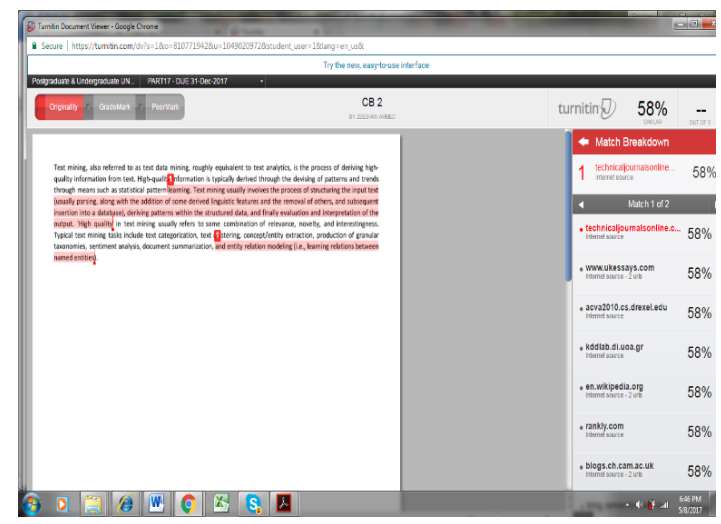

Fig. 9. Problem in Existing System

The fascinating thing is that both documents apparently looks same as shown in Fig. 10.

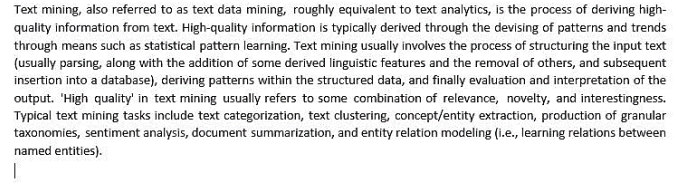

Fig. 10. Apparent Look of submitted Document

But the true matter can be examined in Fig 11. We changed the whole paragraph text colour to red so all character hidden between the words are visible with colour changed to white colour (background colour). By practising this method, cheaters very smartly concatenate various words with illusive character so, they can cheat the plagiarism algorithm.

\section{A. Our System Reports}

The paragraph that contains the illusive/masked text (invisible character between words) is submitted to our system

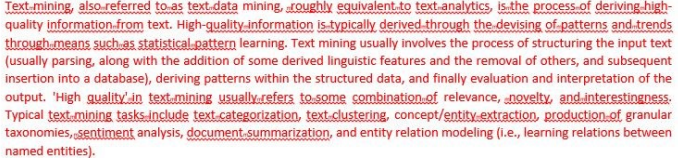

Fig. 11. Illusive text visible in the bypassed document

for checking the plagiarism in the content. But results are remarkable from Turnitin. Our algorithm examines each character colour and ensures that it is visible (colour is distinct from the background). If the algorithm discovers a character which colour is the same as background colour, it replaces that character with the space, and detects that the document is $100 \%$ plagiarised as shown in Fig. 12.

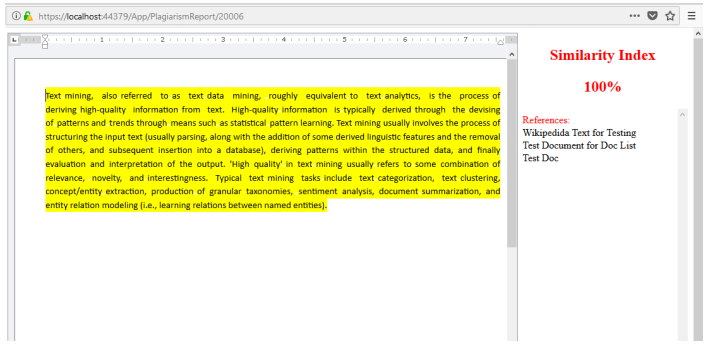

Fig. 12. Our System Output

Another technique that is used by the cheaters to decrease plagiarism rate and increase words count is hiding spam text behind the images. This text is not visible to the evaluator of the document, but it increases the word count. And if the total word increase, eventually plagiarised text count will be reduced, and this will help to reduce the plagiarism percentage of the document. We obtain a document that contains images and garbage text behind it that is not visible to anyone, but it helps to increase the word count as shown in Fig. 13 (report generated using Turnitin).

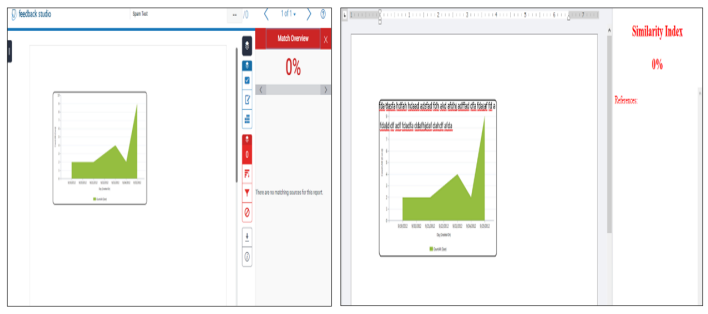

Fig. 13. Turnitin Report with Spam Text in behind(left) and front(right) of Image

As we can see in Fig. 13 (left) text is not visible in the document because text is hidden behind the image. And the plagiarism is $0 \%$ of the document. The document info is shown in Fig. 14 that demonstrate word and character count in the document. 


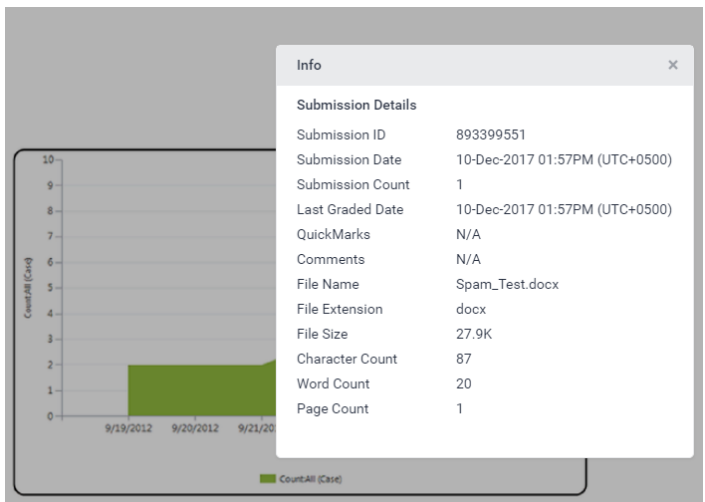

Fig. 14. Document Character Count

But the realistic picture of the document is different. We input the same document (contain garbage text behind the images) to our algorithm. Instead of only counting the spam/garbage text it brings the garbage text to the front of the image so, it is visible to the evaluator of the document as shown in Fig. 13 (right).

\section{CONCLUSION}

Most of the plagiarism detection algorithms check only the content properties (text-colour, image \& text location, etc.) to ensure the validity of the document. In this paper we proposed a solution of two critical problems that are used by the authors (cheater) to bypass the plagiarism detection algorithm i.e. illusive text and text behind images. The cheater smartly changes the document content properties to cheat the algorithm. In order to overcome these loopholes, our proposed algorithm very efficiently handle these two problems.

\section{REFERENCES}

[1] S. Ganguly and G. Veda, "A new randomized algorithm for Document Fingerprinting," Indian Institute of Technology, 2000.

[2] B. Gipp and J. Beel, "Citation Based Plagiarism Detection - A New Approach to Identify Plagiarized Work Language Independently," HT'10, Proceedings of the 21st ACM Conference on Hypertext and Hypermedia, pp. 273-274, 2010.

[3] A. Ekbal, S. Saha and G. Choudhary, "Plagiarism Detection in Text using Vector Space Model," Hybrid Intelligent Systems, 12th International Conference, pp. 363-371, 2012.

[4] S. R. Malik and M. Gulia, "Rabin-Karp Algorithm with Hashing a String Matching tool," International Journal of Advanced Research in Computer Science and Software Engineering, vol. 4, no. 3, pp. 389-392, 2014.

[5] M. L. Brocardo, I. Traore, S. Saad and I. Woungang, "Authorship Verification for Short Messages using Stylometry," International Conference on Computer, Information and Telecommunication Systems (CITS), pp. 1-6, 2013.

[6] S. Schleimer, D. S. Wilkerson and A. Aiken, "Winnowing: Local Algorithms for Document Fingerprinting," International Conference on Management of data, pp. 76-85, 2003.

[7] A. Saini, A. Bahl, S. Kumari and M. Singh, "Plagiarism Checker: Text Mining," International Journal of Computer Applications, vol. 134, 2016.

[8] M. A. C. Jiffriya, A. M. A. C. Jahan and R. G. Ragel, "Plagiarism Detection on Electronic Text Based Assignments using Vector Space Model," in Information and Automation for Sustainability (ICIAfS), Colombo, Sri Lanka, 2014.

[9] A. Si, H. V. Leong and R. W. H. Lau, "A Document Plagiarism Detection System," Symposium on Applied Computing, pp. 70-77, 1997.
[10] Z. Ceska and C. Fox, "The Influence of Text Pre-processing on Plagiarism Detection," RANLP 2009 Organising Committee / ACL, pp. 55-59, 2009.

[11] Asadullah, B. M, I. Stern and V. D. Bhat, "Design Patterns Based Pre-processing of Source Code for Plagiarism Detection," Software Engineering Conference (APSEC), 2012 19th Asia-Pacific, pp. 128-135, 2012.

[12] A. Atayero, A. A. Alatishe and K. O. Sanusi, "Development of iCU: A Plagiarism Detection Software," International Journal of Electrical \& Computer Sciences IJECS-IJENS, vol. 11, no. 4, pp. 30-35, 2011. 